\title{
The Neural Basis of Anxiety Across Menstrual Cycle
}

\author{
Ren-Jen Hwang ${ }^{1,2,3, *}$, Ying-Chia Lin ${ }^{4,5}$, Chi-Hsun $\mathrm{Wu}^{6}$ \\ ${ }^{1}$ Department of Nursing, Chang Gung University of Science and Technology, Taoyuan, Taiwan \\ ${ }^{2}$ Departments of Nursing, Chang Gung Memorial Hospital, Linkou, Taiwan \\ ${ }^{3}$ Center of Clinical Competency Center, Chang Gung University of Science and Technology, Taoyuan, Taiwan \\ ${ }^{4}$ Center for Advanced Imaging Innovation and Research (CAI2R), NYU School of Medicine, New York, USA \\ ${ }^{5}$ Center for Biomedical Imaging, Department of Radiology, NYU School of Medicine, New York, USA \\ ${ }^{6}$ Department of Electrical Engineering, National Central University, Taiwan \\ Email address: \\ rjhuang@mail.cgust.edu.tw (R. J. Hwang), linyingchia@gmail.com (Y. C. Lin), rexchwu@gmail.com (C. H. Wu) \\ ${ }^{*}$ Corresponding author
}

\section{To cite this article:}

Ren-Jen Hwang, Ying-Chia Lin, Chi-Hsun Wu. The Neural Basis of Anxiety Across Menstrual Cycle. International Journal of Psychological and Brain Sciences. Vol. 3, No. 4, 2018, pp. 28-39. doi: 10.11648/j.ijpbs.20180304.11

Received: September 11, 2018; Accepted: September 20, 2018; Published: October 15, 2018

\begin{abstract}
The effects of estrogen on anxiety-like behaviors results are controversial. Menstrual cycle phase modulates anxiety-related neural function in women have not been sufficiently investigated. The neural base of anxiety possible incongruent across menstrual cycle was investigated in the current study. We studied the neural correlates of anxiety across menstrual cycle approach from cortical evoked magnetic field (MEF) activity under threaten cue stimulus by sLORETA in 14 healthy women. Evaluations included comparisons of the time-course, early vs. late processing (EP: 1-250ms; LP: 251-500ms after stimulus onset) during the menstrual (MC) and peri-ovulatory (OV) phases (MC vs. OV), using dynamic spatio-temporal analysis. Healthy women exhibited dissimilar anxiety-associated patterns of fear neuronal circuitry across menstrual cycle. Analyses revealed significant interaction of the time-course (EP vs. LP) and menstrual cycle phase (MC vs. OV) in the highest anxiety-associated regions. Inversely relation of the anxiety state and insular activation was revealed in the MC vs. OV phase. Results indicated that women can use different attention/cognitive resources in response to fear event across the menstrual cycle. This study presents the first evidence that menstrual cycle phase can modulates anxiety- related neural activation in women. Inconsistent anxiety subtypes may occur at different menstrual cycle. These features are an important consideration in understanding the effect of the menstrual cycle on the neural substrates of anxiety, and provide a potential contribute in pathophysiological or therapeutic implications for menstrual cycle-sensitive psychiatric conditions.
\end{abstract}

Keywords: Menstrual Cycle, Anxiety, Brain, Go/NoGo

\section{Introduction}

\subsection{Anxiety in the Menstrual Cycle}

Sex steroids play a key role in the regulation of anxiety and fear. However, the menstrual cycle phase modulates the anxiety-related neural activity in women has not investigated. The effects of estrogen on the anxiety-like behaviors results are controversial. Estradiol decreases anxiety behavior and enhance performance in some cognitive tasks [1-3]. Contrary, excessive estrogen can be produce agitation, irritability and enhances anxiety manifestations as inhibitory avoidance has also document [4-8]. Both hypo- and hyper-estrogenism connect the anxiety related behavior may trace a possibility, that the scenery of anxiety is not a monolithic construct while often overlook manipulated in those hormonal studies. Our observation that the characteristics of anxiety may differ with hormonal status, as well the anxiety provokes hemisphere asymmetry, presenting a resilient affective style across menstrual cycle [9]. Anxiety has specific effects on cognition [10], diverse emotions induced quite different patterns of cognitive processing $[9,11]$ as well the contrasting pattern of brain activity in distinct of anxiety subtypes has report [12]. The present study as extend our previous sensory level analysis [9], evaluating the anxiety associated cortical response to fear cue stimulus at different menstrual cycle. It is 
the first step and preliminary to clarify the neural base of anxiety possible incongruent across menstrual cycle.

\subsection{Threat-Related Attention Bias in Anxious}

Converging evidence has suggested that the attention system of anxious people is particularly sensitive to fear-relevant stimuli [13-15]. Brain attention system is functional segregated by various anxiety in response to threatening cues. Studies have described that exhibiting the 'vigilance" or "avoidance' pattern (capture vs. hold bias) to threaten cues in various anxiety disorders [16]. Typically, a characterized effect of anxiety on cognition is specific increased attention capture by threat-related stimuli [10, 17]. Conversely, fear avoidance or rapid disengagement of attention from negative words demonstrated in social anxiety or panic disorder [18-21]. The different aspects of selective attention in anxiety have yet to be fully elucidated $[14,22]$.

\subsection{Neural Basis of Anxiety}

Neuroimaging studies have demonstrated that brain areas involved in fear circuitry include the hippocampus, amygdala, cingulate, prefrontal and parietal cortex, insula and visual association cortex. Altered insular sensitivity has identified in several clinical populations of anxiety disorders [23]. Converging evidences have suggested that parietal-visual cortical association in the attention system is particularly sensitive to threat cues in anxious people [24, 25]. The parietal cortex plays a critical role in visuospatial processing in response to threats [26, 27], with hemispheric functional lateralization particularly relating to attention selection [28, 29]. The right vs. left posterior parietal cortex (PPC) was associated with the capacity of attention selection bias that relates toward vs. away from salient stimuli had report [29, 30]. rTMS to the right posterior parietal cortex (PPC) disrupted the guidance of attention toward salient stimuli, whereas rTMS to the left PPC affected the ability to bias selection away from salient stimuli [29, 30]. Neuroimage studies have indicated that the anxious apprehension involves more left- than right-frontal activity and that anxious arousal is associated with more right- than left- hemisphere activity $[12,31,32]$. Despite the anxiety take in sensitivity to the attention cue, while has not adequately investigated on the effects specified with menstrual cycle.

\subsection{Dynamic Spatio-temporal Analysis}

The neural network of visual processing is distributed and dynamic. The ERP relies on the assumption of similarity in the activity of interest across trials of the averaging procedure. Previous EEG/MEG studies have implemented wide-ranging time-course analyses [33, 34]. The visual ERP presumes that the time-course of two psychological processes involved in behavioral representations, generally separate as perception vs. cognition, tend to occur in early vs. late processing (EP vs. LP) Generators of the two compare domains lie close to the brain occipital-parietal cortex vs. frontal region, separately. Fear facial NoGo stimulus evokes biological stress responses [35], and adequacy used to elicit negative emotion compared with passively viewing simple unpleasant pictures.

We investigate the neural base of anxiety with menstrual cycle approach by threaten cue challenge due to the fear signal adequate in understanding the physiological and behavioral characteristics of anxiety [32, 36, 37]. Serum estradiol surges significantly during the ovulatory phase [38-40] and reduces at menstruation phase. Evaluations of the anxiety covariates brain activity was included comparing with the time-course, early vs. late processing (EP: 1-250ms; LP: 251-500ms after stimulus onset), and different menstrual cycle, menstruation vs. periovulatory phases (MC vs. OV) by using the standardized low-resolution brain electromagnetic tomography analysis (sLORETA).

\section{Materials and Methods}

\subsection{Participants}

Fourteen right-handed, healthy young women with regular menstrual cycles ( $24-35$ days) were recruited. The sample had an age range of 18 to 35 years. Participants did not use oral/hormonal contraceptives and were not pregnant. The exclusion criteria included (1) a history of neurological or psychiatric disorders or (2) premenstrual syndrome ruled out by the DSM-IV criteria [41]. The subjects were prevented from using caffeine/tobacco for 12 hours, and alcohol for 48 hours before the study. This study was approved by the Institutional Ethics Committee of Taipei Veterans General Hospital. Written informed consent was obtained from each participant prior to starting the study.

\subsection{Procedure}

Each participant underwent two MEG sessions during the menstrual cycle: in the menstrual phase (MC, from the second to the fourth day after menstrual onset) and in the periovulatory phase $(\mathrm{OV}$, from the twelfth to sixteenth day after menstrual onset) as confirmed by the urinary luteinizing hormone (LH) test. The 306-channel MEG system (Vectoview@, Neuromag, Finland)was exploited to measure the brain neuromagnetic activity. The MEG study was conducted within 36 hours of the LH surge, as detected previously [9]. A repeated measurement, counterbalanced design was used to eliminate any ordering effect: $50 \%$ of participants were studied in the MC phase, and the other $50 \%$ were studied first in the OV phase. Each participant completed two measurement sessions within two monthly cycles. Anxiety inventory measurements were assessed following each MEG recording.

\subsubsection{Stimuli}

Participants were required to complete a fear Go/NoGo task. They either responded to a particular emotional facial expression (neutral, sad, happy; Go trials) or were prohibited from responding to a fearful expression (NoGo trials). The stimuli were digitized black and white faces taken from the Ekman collection of faces [42]. Participants saw an image of a 
fixed cross for $500 \mathrm{~ms}$, as a warning signal, followed $1000 \mathrm{~ms}$ later by an image of a facial expression for $400 \mathrm{~ms}$. To discern possible confound contribution from other cognitive component, e.g., executive control, as commonly involved in a Go/NoGo task, subjects also performed an emotionally neutral Go/NoGo task in a different experimental session on the same day. The participant should respond to a symbol set (square, star and triangle; Go trials) but prohibit the response to a circle symbol (NoGo trials). We used the symbol $\mathrm{Go} / \mathrm{NoGo}$ task as the neutral control instead of using neutral face as the NoGo event since the neutral face could be recognized as of negative valence and may further complicate the experimental situation [43, 44]. Pictures (or symbols) were sequentially presented on a white background in the middle of a screen in front of the participant. At least 30 successful NoGo trials were completed for each task (fear and neutral). Participants used the right index finger for the Go response. The frequency ratio of the Go/NoGo trials was $80 \%$ to $20 \%$. The sLORETA procedure was utilized for the successful NoGo trials (fear and neutral).

\subsubsection{MEG Recording}

An anatomical MRI of each participant was acquired. The T1-weight, 3D gradient-echo anatomical MRI was performed on a 3T MR scanner (Bruker, Germany). The matrix size was set to $256 \times 256 \times 128 \mathrm{~mm}^{3}$, and the FOV was $230 \times 230 \times 192$ $\mathrm{mm}^{3}$. Before scanning, the magnetic field shimming was performed automatically, and tri-pilot images were used to adjust the FOV location. Participants were asked to relax and not to move during the scanning procedure.

Participants sat comfortably in a magnetically shielded room. Brain signals were recorded using a whole-head 306-channel neuromagneto- meter (Vectoview, Elekta Neuromag, Helsinki, Finland), digitized at $1024 \mathrm{~Hz}$ using a 0.03 to $330 \mathrm{~Hz}$ band pass filter. Vertical and horizontal electrooculograms were monitored to reject epochs that coincided with blinks and excessive eye movements, with an amplitude cutoff of $600 \mathrm{mV}$. Four head-position-indicator (HPI) coils were attached to each participant's head and were used to ensure that no large head movements occurred throughout the measurement period by comparing the positions of these HPI coils before and after the recordings. To ensure that the different measurements covered the same cortical regions of each participant, three predefined anatomical landmarks (the nasion and bilateral preauricular points) were used to confirm that head positions relative to the sensor array were similar across sessions [45]. The analyzed epoch was from $200 \mathrm{~ms}$ prior to the onset of the stimulus to $1,000 \mathrm{~ms}$ after the onset of the stimulus.

\subsubsection{Behavioral Assessment}

Each participant was requested to complete a State-Trait Anxiety Inventory (STAI) after each MEG measurement, to obtain an index of negative mood. State anxiety (SAI) reflects a transitory emotional state or condition of the human organism [46]. SAI scores were assessed for all participants in the OV and MC phases, respectively. Each of the $20 \mathrm{SAI}$ items was given a weighted score from 1 to 4 , with a rating of 4 indicating the highest level of anxiety. Total score ranged from 20 to 80 .

\subsection{Data Analysis}

\subsubsection{Current Density Reconstruction}

Before implementation of a spatial filtering technique, sLORETA, individual high resolution white matter MRI were conducted. The Brain Extraction Tool (BET tool; part of FSL-FMRIB's Software Library) was used to segment the anatomical MRI, removing the skull and dura from MR images.

Brain activity was reconstructed using Curry 5.0. (Compumedics Ltd., USA) and a spatial filtering technique, sLORETA (standardized low-resolution electromagnetic tomography) was utilized in successful NoGo trials- MEG tomographic analysis. sLORETA as provides a reliable and detailed assessments for quantitative analysis in MEG. The single-dipole model may be insufficient to explain cortical neuromagnetic activity because such activity can have multiple sources. The sLORETA conductor model was used in the current density reconstruction (CDR), and in a modified Minimum Norm Least Squares (MNLS, L2 Norm) approach, calculating the current strength for each location by dividing by its error bar (or the length). The sLORETA is an effective CDR method for resolving the MEG inverse problem. In this study, components of the mean global field power (MGFP) were selected carefully from the evoked related fields (ERF) in response to the fear NoGo stimuli. ERF and MGFP peak analyses were performed across the time window from 1 to $500 \mathrm{~ms}$ following the onset of the stimulus. The coordinates of each channel were transformed through the MRI-MEG integration system. The offline averaged MEG signals were filtered using a $5-30 \mathrm{~Hz}$ band pass filter.

All CDR images were spatially normalized into a standard stereotaxic space using Statistical Parametric Mapping (SPM2; Functional Imaging Laboratory, Wellcome Department of Imaging Neuroscience, UCL, London, UK), and then a $12 \mathrm{~mm}$ Gaussian kernel was used for smoothing for statistical analysis. SPM used the standard brain template developed at the Montreal Neurological Institute (MNI), and the coordinates obtained by SPM were convertible into standard Talairach space.

\subsubsection{Statistical Analysis}

Individual statistical maps of the MGFP were computed for successful NoGo trials in each task (fear and neutral), using the general linear model approach. Two major time windows after stimulus onset: 1 to $250 \mathrm{~ms}, 251$ to $500 \mathrm{~ms}$ was identified early processing (EP) and late processing (LP) by our previous observations [9]. Second-level (group) analysis as random effects model was conducted for statistical group comparisons (MC vs. $\mathrm{OV}$; EP vs. LP). Correlation analysis with an uncorrected threshold $\mathrm{p}$ of.001 (spatial extent threshold $=20$ voxels) was implemented to elucidate the covariate region of anxiety in MC and OV. The highest (rs) correlated region of anxiety score (HCR) in each session (four conditions of MC-EP, MC-LP, OV-EP, OV-LP) as voxel-of-interest (VOI) were selected. Of the four activate regions, was used for clarified the interaction effects of the time-course (EP vs. LP) and menstrual phase (MC vs. OV). 
A two-way repeated measurements of analysis of variance (ANOVA) on HCR was conducted to determine the interaction effect of time-course and menstrual phase using SPSS-12 (SPSS Inc, USA). Pearson correlation was subsequently applied to elucidate the interaction effect in the four HCR regions. The other selected VOI were in the right insula (R Ins) where the HCR was found in OV-LP, because which region plays a key role in anxiety [23]. The VOI of $R$ Ins was extracted with a $6 \mathrm{~mm}$ sphere centering on the region, further allowing phase (MC vs. OV) comparisons. The variance of the behavior data (SAI) and VOI/R Ins for different groups (MC vs. OV) was examined using a paired t-test. The threshold for significance was $p=.05$.

\section{Results}

\subsection{Image Results}

\subsubsection{Brain Activity Modulated by Anxiety in the MC vs. $O V$ Group}

Table 1 shows the neural correlates of anxiety during different time-courses (EP and LP) and menstrual phases (MC vs. OV) during fear NoGo condition. Anxiety covariates cortical activation for MC-EP, primarily in the right parietooccipital regions, including the $\mathrm{R}$ precentral $\mathrm{G}$, sup parietal $\mathrm{L}$, occipital cuneus, and bilateral sup front oparietal G L. Of the MC-LP, anxiety covariates cortical activation primarily in the left temporal- frontal regions, including the left superior frontal gyrus (SFG) [BA6], inferior frontal gyrus (IFG) [BA47], sup temporal G [BA38], and R precentral G [BA4].

The anxiety covariates cortical activation for OV-EP, primarily in the left posterior parietal regions, including $\mathrm{L}$ parietal postcentral $\mathrm{G}$ [BA3/6], inf parietal L [BA40], and R mid-occipital-temporal G [BA19/21]. Of the OV-LP, the anxiety score associated with brain activation primarily in the right insula temporal-frontal cortex (rITFC) areas, including $\mathrm{R}$ insular [BA13], superior temporal gyrus [BA13/39/38/22], inferior/mid frontal G [BA10/46], precentral G [BA6], and left postcentral gyrus [BA5/7].

Table 1. Significant covariates with anxiety score for MC and OV group during fear NoGo.

\begin{tabular}{|c|c|c|c|c|c|c|}
\hline \multirow{2}{*}{ Cortical region } & \multirow{2}{*}{$\mathbf{B A}$} & \multicolumn{3}{|c|}{ Coordinate } & \multicolumn{2}{|c|}{ Voxel level } \\
\hline & & $\mathbf{x}$ & $\mathbf{y}$ & $\mathbf{z}$ & $\mathrm{Z}$ max & $p$ \\
\hline \multicolumn{7}{|l|}{ MC_EC } \\
\hline R Precentral G & 6 & 32 & -12 & 69 & 3.32 & $<0.001$ \\
\hline R Precentral G & 4 & 36 & -29 & 71 & 3.06 & 0.001 \\
\hline R Sup Parietal L & 7 & 20 & -63 & 62 & 3 & 0.001 \\
\hline L Sup Frontal G & 6 & -8 & 0 & 68 & 2.96 & 0.002 \\
\hline L Sup Frontal G & 6 & -4 & 15 & 64 & 2.67 & 0.004 \\
\hline R Sup Frontal G & 6 & 6 & 23 & 62 & 1.7 & 0.045 \\
\hline L Postcentral G & 7 & -8 & -55 & 71 & 2.87 & 0.002 \\
\hline L Precuneus & 7 & -12 & -79 & 56 & 2.2 & 0.014 \\
\hline L Superior Parietal L & 7 & -44 & -65 & 51 & 2.3 & 0.011 \\
\hline R Occipital Cuneus & 19 & 24 & -93 & 40 & 2.13 & 0.016 \\
\hline $\begin{array}{l}\text { R Occipital Cuneus } \\
\text { OV_EC }\end{array}$ & 19 & \multicolumn{4}{|c|}{ OV_EC } & 0.034 \\
\hline L Parietal Postcen G & 3 & -49 & -15 & 54 & 3.2 & 0.001 \\
\hline L Precentral G & 6 & -44 & 0 & 46 & 3.14 & 0.001 \\
\hline L Inf Parietal L & 40 & -40 & -36 & 52 & 2.01 & 0.022 \\
\hline R Mid Frontal G & 6 & 40 & -1 & 61 & 2.77 & 0.003 \\
\hline L Inf Parietal L & 40 & -57 & -50 & 56 & 2.38 & 0.009 \\
\hline R Mid Occipital G & 19 & 59 & -68 & -3 & 1.96 & 0.025 \\
\hline R Mid Occipital G & 19 & 55 & -78 & -6 & 1.74 & 0.041 \\
\hline R Mid Temporal G & 21 & 63 & -60 & 3 & 1.71 & 0.044 \\
\hline R Postcentral G & & 50 & -20 & 58 & 1.91 & 0.028 \\
\hline \multicolumn{7}{|l|}{ MC_LC } \\
\hline L Sup Frontal G & 6 & -14 & 30 & 52 & 3.01 & 0.001 \\
\hline L Sup Frontal G & 6 & -8 & -4 & 67 & 2.96 & 0.002 \\
\hline L Sup Frontal G & 6 & -2 & 23 & 62 & 2.91 & 0.002 \\
\hline L Inf Frontal G & 47 & -40 & 29 & -5 & 2.43 & 0.007 \\
\hline R Precentral G & 4 & 53 & -12 & 39 & 2.4 & 0.008 \\
\hline L Sup Temporal G & 38 & -40 & 13 & -19 & 1.93 & 0.027 \\
\hline L Sup Temporal G & 38 & -49 & 11 & -16 & 1.84 & 0.033 \\
\hline \multicolumn{7}{|l|}{ OV_LC } \\
\hline R Sub-lobar, Insula & 13 & 34 & -42 & 17 & 3.96 & $<0.001$ \\
\hline R Sup Temporal G & 13 & 51 & -40 & 20 & 3.65 & $<0.001$ \\
\hline R Sup Temporal G & 39 & 51 & -53 & 23 & 3.54 & $<0.001$ \\
\hline R Inf Frontal G & 10 & 44 & 49 & 1 & 2.43 & 0.008 \\
\hline R Inf Frontal G & & 55 & 31 & 0 & & \\
\hline R Mid Frontal G & 46 & 44 & 42 & 24 & 2.24 & 0.012 \\
\hline R Precentral G & 6 & 36 & -11 & 58 & 2 & 0.023 \\
\hline R Occipital Cuneus & 18 & 12 & -97 & 12 & 1.97 & 0.024 \\
\hline
\end{tabular}




\begin{tabular}{|c|c|c|c|c|c|c|}
\hline \multirow{2}{*}{ Cortical region } & \multirow{2}{*}{ BA } & \multicolumn{3}{|c|}{ Coordinate } & \multicolumn{2}{|c|}{ Voxel level } \\
\hline & & $\mathbf{x}$ & $\mathbf{y}$ & $\mathbf{z}$ & $\mathrm{Z}$ max $_{\text {max }}$ & $p$ \\
\hline L Postcentral G & 5 & -34 & -43 & 67 & 1.97 & 0.024 \\
\hline L Sup Parietal L & 7 & -18 & -63 & 58 & 1.92 & 0.028 \\
\hline R Paracentral L & 4 & 8 & -36 & 63 & 1.91 & 0.028 \\
\hline R Sup Temporal G & 38 & 55 & 9 & -14 & 1.91 & 0.028 \\
\hline R Sup Temporal G & 22 & 61 & 4 & -4 & 1.85 & 0.032 \\
\hline
\end{tabular}

All voxels are significant at $\mathrm{p}<0.05$, uncorrected for multiple comparisons with extent threshold at 20 voxels; BA: Broman Area; $\mathrm{Z}$ max report the $\mathrm{Z}$ value at the cluster Peak.

Table 2. Significant covariates with anxiety state for MC and OV group during Symbol Neutral NoGo.

\begin{tabular}{|c|c|c|c|c|c|c|}
\hline \multirow{2}{*}{ Cortical region } & \multirow{2}{*}{ BA } & \multicolumn{3}{|c|}{ Coordinate } & \multicolumn{2}{|c|}{ Voxel level } \\
\hline & & $\mathbf{x}$ & $\mathbf{y}$ & $\mathbf{z}$ & $Z_{\max }$ & $p$ \\
\hline \multicolumn{7}{|l|}{ MC_EC } \\
\hline R Middle Frontal G & 10 & 44 & 47 & 14 & 2.72 & 0.005 \\
\hline R Middle Frontal G & 46 & 48 & 43 & 5 & 2.34 & 0.012 \\
\hline R Superior Frontal G & 10 & 36 & 53 & 14 & 2.32 & 0.012 \\
\hline & \multicolumn{5}{|c|}{ OV EC } & 0.007 \\
\hline R Limbic Parahippo G & 36 & 34 & -28 & -24 & 4.07 & $<0.001$ \\
\hline R, Temporal, Fusiform G & 20 & 44 & -32 & -25 & 3.88 & $<0.001$ \\
\hline R Parietal Inferior, G & 40 & 59 & -47 & 39 & 3.66 & $<0.001$ \\
\hline R Superior Frontal G & 10 & 30 & 52 & 23 & 2.54 & 0.006 \\
\hline R Superior Frontal G & 9 & 34 & 46 & 31 & 2.45 & 0.007 \\
\hline R, Occi Cuneus, G & 18 & 4 & -86 & 25 & 2.43 & 0.008 \\
\hline L Parietal Postcentral G & 40 & -57 & -23 & 14 & 2.2 & 0.014 \\
\hline R Occi Cuneus, G & 17 & 10 & -83 & 4 & 2.19 & 0.014 \\
\hline L Occi Lingual G & 17 & -10 & -89 & -2 & 2.08 & 0.019 \\
\hline L Occ Lingual G & 17 & -2 & -85 & 3 & 1.88 & 0.03 \\
\hline R Mid Occi G & 18 & 28 & -93 & 14 & 2.18 & 0.015 \\
\hline R Occi Cuneus & 17 & 20 & -91 & 8 & 1.95 & 0.026 \\
\hline L Sup Temporal G & 22 & -49 & -58 & 14 & 2.11 & 0.018 \\
\hline R Sub-lobar, Thalamus, G & & 10 & -21 & 3 & 2.09 & 0.018 \\
\hline R Sub-lobar, Thalamus, G & & 12 & -25 & 10 & 1.91 & 0.028 \\
\hline L Super Frontal G & 9 & -6 & 58 & 32 & 2.08 & 0.019 \\
\hline L Medial Frontal G & 9 & -24 & 36 & 18 & 2.06 & 0.02 \\
\hline R Middle Occi G & 19 & 50 & -73 & 7 & 2.04 & 0.021 \\
\hline R Middle Occi G & 19 & 48 & -80 & 2 & 1.94 & 0.026 \\
\hline R Middle Temporal G & 39 & 48 & -75 & 15 & 1.92 & 0.027 \\
\hline R Limbic Post Cingulate, $G$ & 30 & 10 & -66 & 11 & 2.01 & 0.022 \\
\hline L Limbic Parahippo G & 35 & -18 & -11 & -25 & 1.97 & 0.024 \\
\hline L Parietal Supramarginal G & 40 & -51 & -49 & 28 & 1.96 & 0.025 \\
\hline $\begin{array}{l}\text { L Temporal, Fusiform G } \\
\text { MC LC }\end{array}$ & 20 & \multicolumn{5}{|c|}{ MC_LC } \\
\hline R Parietal Postcentral G & 2 & 59 & -19 & 47 & 2.86 & 0.002 \\
\hline R Parietal Postcentral G & 2 & 63 & -29 & 42 & 2.56 & 0.005 \\
\hline R Frontal Precentral G & 6 & 59 & -11 & 43 & 2.47 & 0.007 \\
\hline R Sup Parietal G & 7 & 42 & -58 & 49 & 2.77 & 0.003 \\
\hline L Medial Frontal G & 6 & -4 & -14 & 63 & 2.37 & 0.009 \\
\hline R Middle Frontal G & 6 & 38 & -1 & 59 & 2.26 & 0.012 \\
\hline R Frontal Precentral & 6 & 46 & -7 & 56 & 2.08 & 0.019 \\
\hline R Middle Frontal G & 6 & 40 & 7 & 55 & 1.86 & 0.032 \\
\hline $\begin{array}{l}\text { L Inferior Parietal L } \\
\text { OV LC }\end{array}$ & 40 & -38 & -40 & 50 & 1.82 & 0.034 \\
\hline R Mid Occip G & 18 & 34 & -87 & 15 & 3.99 & $<0.001$ \\
\hline R Middle OcciG & 19 & 48 & -79 & 15 & 3.88 & $<0.001$ \\
\hline R Occi Lingual G & 17 & 12 & -85 & 4 & 3.81 & $<0.001$ \\
\hline L Parietal Postcentral G & 40 & -38 & -32 & 51 & 3.63 & $<0.001$ \\
\hline L Temporal G & 42 & -65 & -13 & 12 & 3.26 & 0.001 \\
\hline L Superior Temporal G & 42 & -65 & -25 & 14 & 3.2 & 0.001 \\
\hline R Inferior Frontal G & 9 & 65 & 9 & 25 & 3.51 & $<0.001$ \\
\hline R Mid Frontal G & 46 & 61 & 24 & 23 & 3.11 & 0.001 \\
\hline R Mid Frontal G & 9 & 51 & 25 & 36 & 3.05 & 0.001 \\
\hline R Med Frontal G & 10 & 8 & 47 & 9 & 2.36 & 0.009 \\
\hline $\begin{array}{l}\text { R Sub-lobar Lentiform } \\
\text { NucleusG }\end{array}$ & & 20 & 12 & 9 & 2.07 & 0.019 \\
\hline R Sub-lobar Lentiform & & 20 & 4 & -2 & 1.95 & 0.025 \\
\hline
\end{tabular}




\begin{tabular}{|c|c|c|c|c|c|c|}
\hline \multirow{2}{*}{ Cortical region } & \multirow{2}{*}{ BA } & \multicolumn{2}{|c|}{ Coordinate } & \multirow[b]{2}{*}{$\mathbf{z}$} & \multicolumn{2}{|c|}{ Voxel level } \\
\hline & & $\mathbf{x}$ & $\mathbf{y}$ & & $\mathrm{Z} \max$ & $p$ \\
\hline \multicolumn{7}{|l|}{ NucleusG } \\
\hline R Sub-lobar CaudateG & & 14 & 17 & -8 & 1.77 & 0.039 \\
\hline R Frontal Precentral G & 4 & 32 & -24 & 62 & 1.91 & 0.028 \\
\hline R Parietal Postcentral G & 3 & 24 & -30 & 66 & 1.69 & 0.045 \\
\hline
\end{tabular}

All voxels are significant at $\mathrm{p}<0.05$, uncorrected for multiple comparisons with extent threshold at 20 voxels

\subsubsection{Comparing Spatiotemporal Processing for $\mathrm{MC}$ vs. $\mathrm{OV}$}

The four HCRs of anxiety- neurocircuit exhibited (MC-EP, MC-LP, OV-EP, OV-LP) a significant interaction for the time-course (EP vs. LP) and menstrual phase (MC vs. OV) $[\mathrm{F}$ $(1,11)=33.21 ; p<.0001]$. Significant greater activation in the LP during the MC phase (EP $<\mathrm{LP} ; p=.001)$ and contrary, greater activation in the EP in the $\mathrm{OV}$ group $(\mathrm{EP}>\mathrm{LP} ; p=.002)$ (Figure 1). It displays that the anxiety covariates discrepancy cognitive resources across menstrual phase. The MC group used more late than early processing, whereas the OV group used more early than late processing in response to fear cues. Comparing different menstrual phase, we found a sub-significant change that the HCR activation of EP greater in $\mathrm{OV}$, while the activation of LP greater in the MC (EP: $\mathrm{MC}<\mathrm{OV} / p=0.068$; LP: $\mathrm{MC}>\mathrm{OV} / p=0.121$ ).

The four HCRs of anxiety- neurocircuit exhibited inverse lateralization patterns (as hemispheric transfer) during different time-courses (EP vs. LP) and menstrual cycles (MC vs. OV) for fear NoGo condition. The pattern of hemispheric transition through the time-course, EP to LP was found the left- then right- hemispheric processing in the MC phase and the right- then left- hemisphere processing refers to the $\mathrm{OV}$ phase (Table 1). There was no intra- and inter-hemispheric shift observed in the emotionally neutral NoGo trial from this dynamic covariate analysis (Table 2).

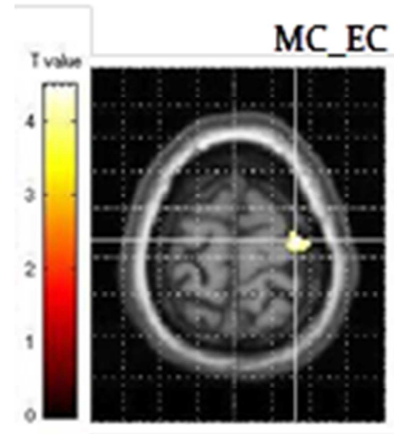

$\mathrm{z}=74 \mathrm{~mm}$

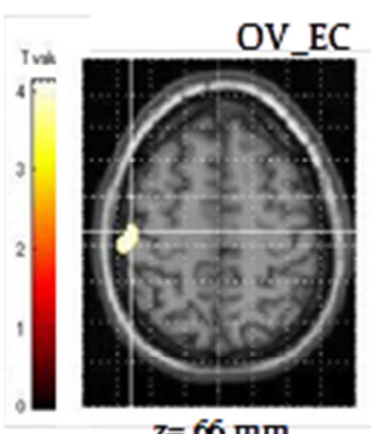

$z=66 \mathrm{~mm}$

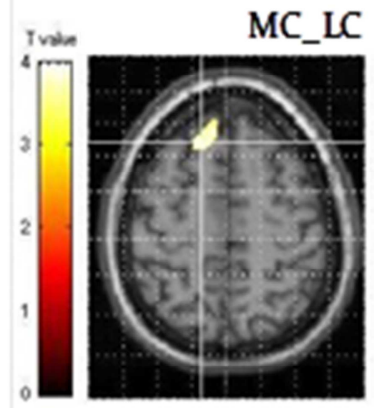

$\mathrm{z}=66 \mathrm{~mm}$

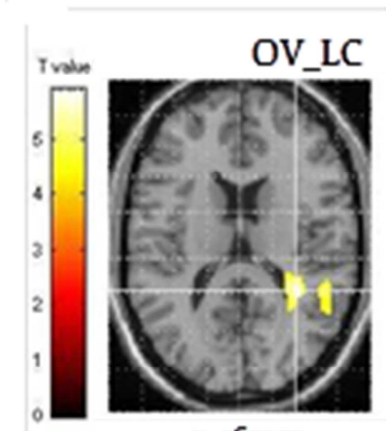

$z=16 \mathrm{~mm}$

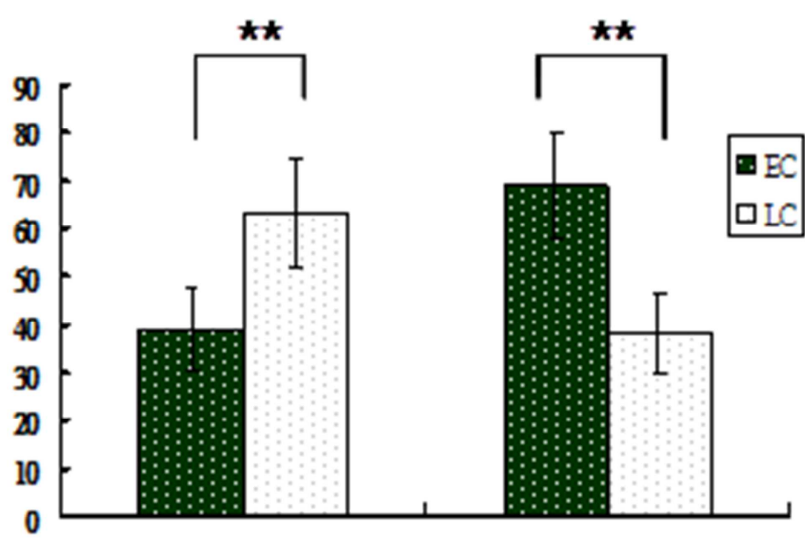

mc

ov

Figure 1. Slice display the brain regions correlated with anxiety score at different time course during fear NoGo stimuli across menstrual cycle. The highest correlated areas of anxiety show interhemispheric transfer include: (1) R FPG for MC_EC; (2) L SFG for MC_LC; (3) L PPC for OV_EC; (4) R Insular for OV_LC. Significant interaction of the time course (EC vs. LC) with menstrual phase (MC vs. OV) was revealed (F $=33.21 ; P<0.001)$. The Bar displayed a contrast draft of activation strength in 4 regions: $M C$ : $E C<L C ; O V: E C>L C$ (both $P<0.005$ ). All voxels are significant at $p<0.001$, uncorrected for multiple comparisons with extent threshold at 20 voxels. L: left; R: right; EC: early component; LC: late component; PPC: posterior parietal cortex. The dynamics hemispheric specialization integrates in the context by the 4 highest anxiety covariate regions was exhibit.

\subsection{3. $\mathrm{ROI}$}

The HCR of the right insula $[34,-42,17]$ (R Ins) in OV-LP (Table 1) was specific selected for further comparing in MC vs. $\mathrm{OV}$. Inversely relation of the anxiety score and insular was found in fear NoGo condition across menstrual cycle (Figure 2). Significant negative vs. positive relation was occurred in the MC vs. OV respectively $(r=-0.697 ; r=+0.862$; both $p$ $<.05$ ) (Figure 2). 

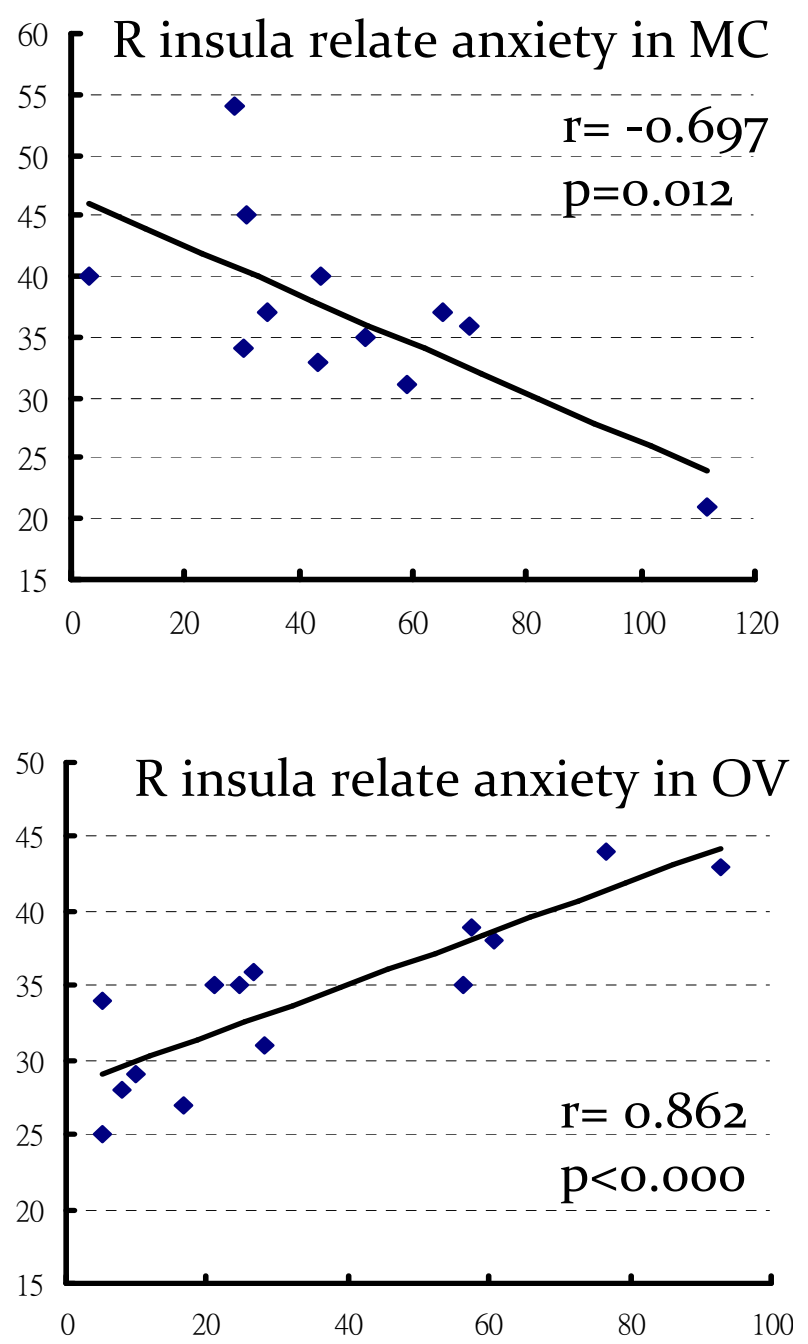

Figure 2. The anxiety scores were inversely related to insular [34,-42, 17] across menstrual cycle phase. Significant negative vs. positive relationships exhibited in the MC vs. OV phases, respectively (both $p<0.001$ ).

\subsection{Psychological Assessment}

There was no difference in anxiety score (SAI) between the menstrual phases. The SAI mean score \pm standard deviation (SD) was 35.8 \pm 8.4 and 34.2 \pm 5.7 during the MC and OV phase respectively. There was no difference in the error rate between fear facial NoGo trail (17\% for MC vs. $18 \%$ for OV) and neutral symbol NoGo trial ( $3 \%$ for MC vs. $4 \%$ for OV) across the menstrual cycle. The error rate for fear facial NoGo trials did not differ between the menstrual phases $(17 \%$ for $\mathrm{MC}$ vs. $18 \%$ for OV).

\section{Discussion}

This study investigates the neural base of anxiety by evaluating cortical evoked magnetic field (MEF) activity in response to fear cues across menstrual cycle. Evaluations of anxiety covariates brain activity included comparisons of the time-course (EP: 1-250ms; LP: 251-500ms after stimulus onset) and different menstrual cycle (MC vs. OV) in a dynamic spatio-temporal analysis. There were two main findings: (1) women show dissimilar anxiety-associated patterns in neuronal activation of fear circuitry in different menstrual cycle. Significant interaction between the time course (EP vs. LP) and menstrual cycle (MC vs. OV) was revealed in the region of the highest anxiety covariates regions. Inter-hemispheric transfer from the EP to the LP occurred only during the fear NoGo condition; and (2) significant negative vs. positive relationships of the anxiety score and insula activation was exhibited in the MC vs. OV phases, respectively (both $p<.05$; Figure 2). Images and behavioral studies have revealed that the threaten signal as an important role in modulating spatial attention in anxiety [10, 17, 25]. Dynamic transfer of the intra- and inter-hemisphere processing to fear cue implied that women can utilize different attention/cognitive resources in their different menstrual cycle. Different emotions are characterized by distinct patterns in cognitive processing [11], and thus, inconsistent anxiety subtypes may exist in different menstrual cycle phases.

\subsection{General View}

Anxiety-associated patterns in neuronal activation of fear circuitry were dissimilar across the menstrual cycle. Significant interaction between the time-course (EP vs. LP) and menstrual cycle (MC vs. OV) was evident in the four HCRs (highest $r_{\mathrm{s}}$ correlated region with anxiety score) ( $p$ $<.0005$ ). Anxiety recruited more cognitive (or action) neural substrate in the MC phase, whereas the OV period favored the perception domain (MC: EP < LP, $p=.001$; OV: EP > LP, $p$ $=.002$ ). Estrogen enhances prefrontal-related cognitive function [11], relative lower estrogen is associated with well documented deficits in high-order cognitive processes. High load on „frontal ${ }^{\text {ee }}$ cognitive control processes increased may illustrate a compensation mechanism during female $\mathrm{MC}$ phase. Females with Turner's syndrome, lacking estrogen due to genetic deficit, could compensate for executive dysfunction via recruitment of additional prefrontal cortex regions involved in inhibition, attention, and working memory for successful performance of Go/NoGo tasks [47]. Conversely, female present significant activation in the perception- neural substrate in OV phase. The perception process reinforcements empirical relates to motivational force. Female may have more perceptive to fear stimuli, warning the organism of possible impending danger in the OV phase. The high perceptual load whereas might provide an optimal condition succeeding for their propagation.

The neural correlates of the anxiety exhibited spatiotemporal dynamic transfer across the menstrual cycle. Interhemispheric transmission throughout the time-course showed marked variations, MC: right to left and OV: left to right during the fear NoGo condition, but not in neutral condition (Table 1, 2). The interhemispheric integration for lateralized cognitive processes was reported by hormonedependent modulation [48-50]. A powerful neuromodulatory action of estradiol on the dynamics of functional brain organization in the female brain had reported [50]. The LH dominances to the activation regulatory system while the $\mathrm{RH}$ device to the arousal regulatory system. It conjectured to determine hemispheric specialization for perception, action, 
emotion and cognition [51]. Interaction between the cerebral hemispheres may allow both hemispheres to contribute their processing resources in order to cope efficiently with complex tasks [52]. Dysfunctional hemispheric transformation signature an index of neuropsychiatry ill-health base has demonstrated [29, 53-56]. These features are an important consideration in understanding that the dynamics of neurons or brain areas and how their interactions of anxiety with hormonal modulation are influenced possibility by and shape behavior. Estradiol can modulate functional brain organization [50], results further indicated that the Anxiety as a vigor moderator integrate in the intra- and inter-hemispheric dynamic transformation and regulating intrinsically self organization to fear cue response across menstrual cycle.

\subsection{Specific View}

\subsubsection{Anxiety Covariates Brain Activity in MC-EP}

Anxiety covariates with neural circuitry was primarily in the right hemisphere, including the occipital cuneus [BA19], superior parietal lobe [BA 7], and frontal-cingulate gyrus (FCG) networks [BA4, 6]. The parieto-occipital area (PO) of the right side is known involving basic visual processing. Visual association of the extrastriate cortices [BA 18, 19] was related to anxiety mood modulation and threaten cue detected [28]. Previous studies have shown that responses to danger signals in the visual cortex associated with an adaptation mechanism for rapid discrimination to aversive stimuli [10]. Numerous reports have described the anxiety can be modulating the activation of the right visual-parietal network [10]. Observations of the right posterior parietal cortex (rPPC) [BA7] were specifically relating the behavior of engage attention cue in normal or anxious participants [10]. Results indicate that the anxiety engaged relative sensitivity on the part of the visual-parietal network of right hemispheric functional in danger cue attend/approach [57] to fear cue early processing for MC phase.

\subsubsection{Anxiety Covariates Brain Activity in MC-LP}

Anxiety associated with neuron activation was primarily in the left hemisphere, including the SFG [BA6] and part of the dorsal lateral prefrontal cortex (dlPFC), during MC-LP. Prior research identified left dlPFC activation in the cognitive model of anxiety [10]. This area specializes in attentionalcognitive functions [58] with involvement in the performance of cognitive demands of anxious apprehension [12] in trait-anxious people [17]. Results concerning the left side of the SFC、LSTG and OFG suggest a specific reward bias function [59, 60], particularly in the OFG regions [BA 47], which are associated with inhibitory control processes [61], in which reward value plays a mediating role. Control over fear/stressors is a critical determinant of a person's physical and psychological wellbeing [62], with control of attention and receptiveness to emotionally evocative stimuli is a basic form of emotional regulation [63]. Anxiety in the MC condition involves the reward stream of the OFC, which may reveal a natural require for mediating subjective hedonic experience [64].

\subsubsection{Anxiety Covariates Brain Activity in $\mathrm{OV}-\mathrm{EP}$}

The neural components of anxiety in the OV phase was included the left PPC and parietal-temporal regions (Table 1). The left PPC has an important role in ignoring danger or grasping action in response to an attentional cue [29, 65]. Serial TMS and neuroimaging studies support that the bilateral PPC has opposite bias in the selection or suppression of saliency. The right vs. left PPC was associated with toward vs. away salient stimuli, as described [29, 30]. In previous studies, activation of the left intraparietal sulcus (IPS; BA40) associated with motor attention [66-68] and visually guided grasping $[69,70]$ in response to salient stimuli. The IPCC system is involved in saliency cue suppression, with relevance to the neuropsychological findings that several anxiety disorders exhibit hold or disengage components of visual attention. Studies have described patients with anxiety and phobia as displaying fear avoidance of threat-related expressions [14, 16, 22], and showing behavioral inhibition or freezing $[71,72]$. The administration of estrogen can enhance freeze-avoidance responses to danger cues in ovarectomized mice [7]. Cortical activation of the IPCC network which covarying with anxiety score in OV-EP, might provide rapid protection against potential harm and contribute in propagation for the species.

\subsubsection{Anxiety Covariates Brain Activity in $\mathrm{OV}-\mathrm{LP}$}

Significant anxiety correlated brain regions were turning to the right insular temporal-frontal cortex (rITFC) areas in OV-LP (Table 1). The rITFC network is involved in the dorsal attention system, and related hypervigilance, sensitivity to anxious arousal [12], panic, phobias, and non-remarkable anxiety ratings in participants [23]. Functional neuroimaging or lesion studies have reported that the brain activation of anxious arousal specifically occurring in the right side, including the dlPFC, vlPFC, and vmPFC [73-79]. HRT can enhance activation of the right hemispheric vigilance system in postmenopausal women [80]. Estrogenic effects on fear and arousal status mostly arise in the limbic-amygdala structures [8], which reciprocally connect to the lateral intraparietal area (LIP) [BA 7], and critically support avoidance-related behaviors. Prior researches have suggested that the right insula has an important role in the anticipation of affective processing during aversive images, and that anticipation of future harm is a key aspect of anxiety [81, 82]. The right inferior PFC (rIPFC), which forms part of the vlPFC, specializes in inhibition of negative emotions or inappropriate motor responses [83-86]. Regions may afford a capacity for obliterate the left PPC create in EC, may well neutralize ongoing withdraw responses reinforcement, outlines a possible that minimize the potential of social rejection and maintain safety behaviors in the OV phase

\subsection{ROI: Relationship Among Anxiety and Insula Activation}

We also confirmed in our brain regions of interest significant relationships with anxiety score and the posterior insula, which reversed directions as significant positive vs. 
negative correlation disclosed in OV $(\mathrm{r}=+0.862 ; p<.001$; $)$ vs. MC phase respectively $(r=-0.697, p<.05)$ (Figure 2$)$. The region of the insula is critical for cognitive, affective, and interoceptive state processes, and associate with attention property. The posterior insula is involved in the integration of excitatory and inhibitory neurotransmission associated with BDNF, which can regulate stress and anxiety-like behaviors $[87,88]$. Altered insular sensitivity has been observed in several clinical anxiety disorders (not all) which hyperactivity as a neuroimage marker of anxiety proneness [23, 82, 89]. Paulus [23] has reviewed that individuals who are prone to anxiety show an altered interoceptive prediction signal, i.e., manifest augmented detection of the difference between the observed and expected body state [81, 82]. Women may have a higher awareness on the internal state of their bodies, thus activate the posterior insula, of engage region attention resources to modulate cognitive responses or behavior in the OV phase.

The right insula plays a major role in diverse cortical functions as well in cardiac autonomic control [90, 91]. It is associated with arousal and sympathoadrenal stress responses and also involved in parasympathetic functions, which engages diverse homeostatic afferents for asymmetrical lateralization in the left and right forebrain had documented [92, 93]. Previous studies reported that estrogen excites neurons of the insula cortex by modulating GABA neurotransmission [94], enhancing its sympatho- excitatory effects [95]. Asymmetric homeostatic afferent activation of the insular and evoke opponent hemispheric processing to anxiety state simultaneously observed in the hormonal modulation, resonance the scenery of anxiety driving diverse in the MC vs. OV. The anxiety score negative relates to insular may account a down-regulating activity of the posterior insula as by relay more left SFC and IFC neuron engagement (Table 1) for possibility varies of perception of the internal body state or emotional regulation during MC phase. Inversely relation of the anxiety state and insular except response to the anxiety motivating incongruent at $\mathrm{MC}$ vs. $\mathrm{OV}$, also reverberate the role of the insular in play a diverse functions, linked to emotion or the regulation of the body's homeostasis at different menstrual phase.

\subsection{Converge Summary}

The neural base of anxiety possible incongruent across menstrual cycle was investigated in the current study. Result disclosed that anxiety associated with dissimilar fear neurocircuitry during different female menstrual phases. Anxiety covariates cortical activation revealed a dynamic intra- and inter-hemisphere interaction in the occipital -parietal-frontal association, that account female can utilize different cognitive resources in response to fear cue across menstrual cycle. Base on the anxiety has specific effects on cognition, different emotions are characterized by distinct patterns in cognitive processing [11, 14]. We claimed that inconsistent anxiety subtypes may occur at different stages of the menstrual cycle. In spite of the author Heller et al. have report that the anxious apprehension involves more left- than right-frontal activity and that anxious arousal is associated with more right- than left- hemisphere activity [12, 31, 32], the anxious apprehension (e.g., worry) and anxious arousal (e.g., panic) whether mutually exclusive precisely within both phase (MC vs. OV) needs to be tested experimentally in the future. These data may offers an interpreted for the effects of estrus status on anxiety related behaviors have not arrived at a clear consensus, that may originate by ambiguous measure in past hormonal research as of the scenery of anxiety is not a monolithic construct. Our result perspective to the neural basis of anxiety can be modulating by female hormonal cycle, cleverly utilize an identical inventory in dynamic relative comparison analysis. Results suggesting the types of anxiety should be considerate into experimental manipulations in future hormonal research, as well significantly differ in psychological and physical characteristic could present in subtypes of anxiety.

The present findings are tempered by several limitations. First, there are several features of the fear NoGo study could be deliberate, like the result also suitable claimed in an opponent appetitive-aversive neural processes or affect regulatory processes to negative emotional challenge. While the main goal of study address the anxiety-neural base with menstrual cycle modulation in virtue of our previous sensor level report [9]. Hence, findings of hemispheric lateralized in PPC and PFC in each EP vs. LP observed allow us debate those network towards in threat-related responses of cognitive biases, based on attention system of anxious individuals particularly sensitive to the fear event. Second, the results are based on relatively small samples, while study conduct a within-subject comparison design which ameliorate inter-subject variations, thus yield a higher statistical power than that of between-subject comparison experiment [48].

It is known that natural fluctuation of sex hormone levels during the menstrual cycle, i.e. estrogen in particular, can modulate functional cerebral asymmetries and the interhemispheric crosstalk [50]. The self-organization process can be task specific which are dependent on cognitive, emotional and behavioral element as well as biological demand at different menstrual cycle. The effect of the menstrual cycle on the neural substrates of anxiety exhibited dissimilar patterns of fear response across menstrual cycle, optimize for a potential contribute provided in pathophysiological or therapeutic implications for menstrual cyclesensitive psychiatric conditions.

\section{Conclusion}

The study investigated the possible incongruent of the neural basis of anxiety across menstrual cycle. Dissimilar anxiety-associated patterns of neuronal activation in response to fear stimuli were found at different menstrual phase. Anxiety covariates cortical activation revealed a dynamic intra- and inter-hemisphere interaction in the occipital -parietal-frontal association, account that female can utilize different cognitive resources in response to fear cue across menstrual cycle. This study presents the first evidence that the 
menstrual cycle phase can modulates anxiety-related neural activation in women. Inconsistent anxiety subtypes may occur at different menstrual cycle. Future investigation of the precise anxiety subtypes exhibited during different menstrual cycle phases is warranted.

\section{Acknowledgements}

The authors would like to thank Chih-Che Chou and Chou-Ming Tseng for MEG technical support. In addition, we would like to thank IBRU of our front line colleagues for their consultation and Dr. Jen-Chuen Hsieh for stimulating discussions on many issues, continued support and invaluable contributions. Data was collected in 2008 and completely analyzed in 2013. The main funding of study was sponsored by the National Science Council (97 - 2314 - B - 010 005).Thanks to economy sponsorship by Chang Gung Memorial Hospital (BMRP C52), (MOST 103-2410-H-255001) supply for statistic consult, document editing and successive publication. The authors declare that they have no competing interests. We also sincerely appreciate the editor's patience in reviewing this article.

\section{References}

[1] JC A: The female brain hypoestrogenic continuum from the premenstrual syndrome to menopause. A hypothesis and review of supporting data 1996, J Reprod Med 41ap:633-639.

[2] BB S: Estrogen and cognitive functioning in women. Proc Soc Exp Biol Med 1998, 217:17-22.

[3] Walf AA FC: Estradiol decreases anxiety behavior and enhances inhibitory avoidance and gestational stress produces opposite effects. Stress 2007, 10:251-260.

[4] Warren MP B-GJ: Mood and behavior at adolescence: evidence for hormonal factors. J Clin Endocrinol Metab 1989, 69:77-83.

[5] Schmidt PJ NL, Danaceau MA, Adams LF, Rubinow DR: Differential behavioral effects of gonadal steroids in women with and in those without premenstrual syndrome. N Engl J Med 1998, 338:209-216.

[6] Morgan MAPD: Effects of estrogen on activity and fear-related behaviors in mice. Horm Behav 2001, 40:472-482.

[7] Morgan MA PD: Estrogen's effects on activity, anxiety, and fear in two mouse strains. Behav Brain Res 2002, 132:85-93.

[8] Morgan MA SJ, Pfaff DW: Estrogens and non-reproductive behaviors related to activity and fear. Neurosci Biobehav Rev 2004, 28:55-63.

[9] Hwang RJ WC, Chen LF, Yeh TC, Hsieh JC Female menstrual phases modulate human prefrontal asymmetry: a magnetoencephalographic study. Horm Behav 2009, 55:203-209.

[10] SJ B: Neurocognitive mechanisms of anxiety: an integrative account. Trends Cogn Sci 2007, 11:307-316.

[11] Maxwell JS DR: Emotion as motion: asymmetries in approach and avoidant actions. Psychol Sci 2007, 18:1113-1119.
[12] Nitschke JB HW, Palmieri PA, Miller GA: Contrasting patterns of brain activity in anxious apprehension and anxious arousal. Psychophysiology 1999, 36:628-637.

[13] Egloff B H, M.: Interactive effects of state anxiety and trait anxiety on emotional Stroop interference. Personality and Individual Differences 2001, 31:875-882.

[14] Fox E RR, Bowles R, Dutton K: Do threatening stimuli draw or hold visual attention in subclinical anxiety? J Exp Psychol Gen 2001, 130:681-700.

[15] Nitschke JB HW: Distinguishing neural substrates of heterogeneity among anxiety disorders. Int Rev Neurobiol 2005, 67:1-42.

[16] Mogg K BB, de Bono J, Painter M: Time course of attentional bias for threat information in non-clinical anxiety. Behav Res Ther 1997, 35:297-303.

[17] SJ B: Trait anxiety and impoverished prefrontal control of attention. Nat Neurosci 2009, 12:92-98.

[18] Derryberry D RM: Temperament and attention: orienting toward and away from positive and negative signals. J Pers Soc Psychol 1994, 66:1128-1139.

[19] Lang PJ BM, Fitzsimmons JR, Cuthbert BN, Scott JD, Moulder B, Nangia V: Emotional arousal and activation of the visual cortex: an fMRI analysis. Psychophysiology 1998, 35:199-210.

[20] Horley K WL, Gonsalvez C, Gordon E: Social phobics do not see eye to eye: a visual scanpath study of emotional expression processing. J Anxiety Disord 2003, 17:33-44.

[21] Bar-Haim Y LD, Pergamin L, Bakermans-Kranenburg MJ, van IMH: Threat-related attentional bias in anxious and nonanxious individuals: a meta-analytic study. Psychol Bull 2007, 133:1-24.

[22] Koster EH CG, Van Damme S, Verschuere B, De Houwer J: Does imminent threat capture and hold attention? Emotion 2004, 4:312-317.

[23] Paulus MP SM: An insular view of anxiety. Biol Psychiatry 2006, 60:383-387.

[24] Rushworth MF PT, Sipila PK Attention systems and the organization of the human parietal cortex. J Neurosci 2001b, 21:5262-5271.

[25] JD B: Brain imaging in anxiety disorders. Expert Rev Neurother 2004, 4:275-284.

[26] Pardo JV FP, Raichle ME: Localization of a human system for sustained attention by positron emission tomography. Nature 1991, 349:61-64.

[27] Jonides J SE, Koeppe RA, Awh E, Minoshima S, Mintun MA: Spatial working memory in humans as revealed by PET. Nature 1993, 363:623-625.

[28] Corbetta M SG: Control of goal-directed and stimulus-driven attention in the brain. Nat Rev Neurosci 2002, 3:201-215.

[29] Mevorach C HG, Shalev L: Opposite biases in salience-based selection for the left and right posterior parietal cortex. Nat Neurosci 2006, 9:740-742.

[30] Mevorach C HG, Shalev L: Reflexive and preparatory selection and suppression of salient information in the right and left posterior parietal cortex. J Cogn Neurosci 2009, 21:1204-1214. 
[31] Heller W NJ, Etienne MA, Miller GA: Patterns of regional brain activity differentiate types of anxiety. J Abnorm Psychol 1997, 106:376-385.

[32] Engels AS HW, Mohanty A, Herrington JD, Banich MT, Webb AG, Miller GA: Specificity of regional brain activity in anxiety types during emotion processing. Psychophysiology 2007, 44:352-363.

[33] Posner MI SB, Odludas Y, Tang Y: Analyzing and shaping human attentional networks. Neural Netw 2006, 19:1422-1429.

[34] Musizza B SA, McClintock PV, Palus M, Petrovcic J, Ribaric S Bajrovic FF: Interactions between cardiac, respiratory and EEG-delta oscillations in rats during anaesthesia. J Physiol 2007, 580:315-326.

[35] Lerner JS DR, Hariri AR, Taylor SE: Facial expressions of emotion reveal neuroendocrine and cardiovascular stress responses. Biol Psychiatry 2007, 61:253-260.

[36] Williams JM MA, MacLeod C: The emotional Stroop task and psychopathology. Psychol Bull 1996, 120:3-24.

[37] Cannistraro PA RS: Neural circuitry of anxiety: evidence from structural and functional neuroimaging studies. Psychopharmacol Bull 2003, 37:8-25.

[38] Krug R PW, Fehm HL, Born J: Selective influence of the menstrual cycle on perception of stimuli with reproductive significance: an event-related potential study. Psychophysiology 2000, 37:111-122.

[39] O'Reilly MA CC, Lawlor BA, Walsh CD, Rowan MJ: The effect of the menstrual cycle on electrophysiological and behavioral measures of memory and mood. Psychophysiology 2004, 41:592-603.

[40] Pearson R LM: Fear recognition across the menstrual cycle. Horm Behav 2005, 47:267-271.

[41] AP A: Diagnostic and Statistical Manual of Mental Disorders (4th ed ed), vol. Washington. DC; 2000.

[42] Ekman PaF WV: Pictures of Facial Affect [slides] Consulting Psychologists Press. Palo Alto, CA, USA,; 1976.

[43] Casey BJ CF, Giedd JN, Marsh WL, Hamburger SD, Schubert $A B$, Vauss YC, Vaituzis AC, Dickstein DP, Sarfatti SE, Rapoport JL: Implication of right frontostriatal circuitry in response inhibition and attention-deficit hyperactivity disorder. J Am Acad Child Adolesc Psychiatry 1997, 36:374-383.

[44] Chiu Y-C L, C.-H., Lin, J.-H,: The neurtral face is not really expressionless and emotionless. Taiwanese Psychology Association 46th Annual Conference 2007:35.

[45] Hwang RJ CL, Yeh TC, Tu PC, Tu CH, Hsieh JC: The resting frontal alpha asymmetry across the menstrual cycle: a magnetoencephalographic study Hormones and behavior 2008, 54:28-33.

[46] Spielberger CD G, Richard L., and Lushene, Robert E.: Manual for the State-Trait Anxiety Inventory (Form Y). Palo Alto: Consulting Psychologist Press; 1983.

[47] Tamm L MV, Reiss AL: Abnormal prefrontal cortex function during response inhibition in Turner syndrome: functional magnetic resonance imaging evidence. Biol Psychiatry 2003, $53: 107-111$
[48] Compton RJ CC, Diepold J: Interhemispheric integration during the menstrual cycle:failure to confirm progesterone-mediated interhemispheric decoupling. Neuropsychologia 2004, 42:1496-1503.

[49] Bayer U KN, Gunturkun O, Hausmann M: Interhemispheric interaction during the menstrual cycle. Neuropsychologia 2008, 46:2415-2422.

[50] Weis S HM, Stoffers B, Vohn R, Kellermann T, Sturm W Estradiol modulates functional brain organization during the menstrual cycle: an analysis of interhemispheric inhibition. J Neurosci 2008, 28:13401-13410.

[51] E Z: Brain Asymmetry, vol. Ed: International Encyclopedia of the Social \& Behavioral Sciences 2: Amsterdam;New York: Elsevier; 2001.

[52] MT B: The missing link: the role of interhemispheric interaction in attentional processing. Brain Cogn 1998, 36:128-157.

[53] TJ C: Schizophrenia as failure of hemispheric dominance for language. Trends Neurosci 1997, 20:339-343.

[54] Fallgatter AJ SW: Reduced frontal functional asymmetry in schizophrenia during a cued continuous performance test assessed with near-infrared spectroscopy. Schizophr Bull 2000, 26:913-919.

[55] Endrass T MB, Rockstroh B: Reduced interhemispheric transmission in schizophrenia patients: evidence from event-related potentials. Neurosci Lett 2002, 320:57-60.

[56] Koch G RJ: TMS investigations into the task-dependent functional interplay between human posterior parietal and motor cortex. Behav Brain Res 2009, 202:147-152.

[57] Kanwisher N WE: Visual attention: insights from brain imaging. Nat Rev Neurosci 2000, 1:91-100.

[58] Yamasaki H LK, McCarthy G: Dissociable prefrontal brain systems for attention and emotion. Proc Natl Acad Sci U S A 2002, 99:11447-11451.

[59] Pochon JB LR, Fossati P, Lehericy S, Poline JB, Pillon B, Le Bihan D, Dubois B: The neural system that bridges reward and cognition in humans: an fMRI study. Proc Natl Acad Sci U S A 2002, 99:5669-5674.

[60] Pizzagalli DA SR, Henriques JB, Davidson RJ Frontal brain asymmetry and reward responsiveness: a source-localization study. Psychol Sci 2005, 16:805-813.

[61] Miller EK CJ: An integrative theory of prefrontal cortex function. Annu Rev Neurosci 2001, 24:167-202.

[62] Salomons TV JT, Backonja MM, Shackman AJ, Davidson RJ: Individual differences in the effects of perceived controllability on pain perception: critical role of the prefrontal cortex. J Cogn Neurosci 2007, 19:993-1003.

[63] Ochsner KN GJ: The cognitive control of emotion. Trends Cogn Sci 2005, 9:242-249.

[64] ML K: The human orbitofrontal cortex: linking reward to hedonic experience. Nat Rev Neurosci 2005, 6:691-702.

[65] Drew AS vDP: The contribution of the human PPC to the orienting of visuospatial attention during smooth pursuit. Exp Brain Res 2007, 179:65-73. 
[66] Rushworth MF KM, Passingham RE The attentional role of the left parietal cortex: the distinct lateralization and localization of motor attention in the human brain. J Cogn Neurosci 2001a, 13:698-710.

[67] Rushworth MF J-BH, Gobel SM, Devlin JT: he left parietal and premotor cortices: motor attention and selection. T Neuroimage 2003, 20 Suppl 1:S89-100.

[68] Jubault T OC, Koechlin E: Serial organization of human behavior in the inferior parietal cortex. J Neurosci 2007 , 27:11028-11036.

[69] Duncan J SR, Kolodny J, Bor D, Herzog H, Ahmed A, Newell FN, Emslie H: A neural basis for general intelligence. Science 2000, 289:457-460.

[70] Culham JC DS, DeSouza JF, Gati JS, Menon RS, Goodale MA: Visually guided grasping produces fMRI activation in dorsal but not ventral stream brain areas. Exp Brain Res 2003, 153:180-189.

[71] Kalin NH SS, Fox AS, Oakes TR, Davidson RJ: Brain regions associated with the expression and contextual regulation of anxiety in primates. Biol Psychiatry 2005, 58:796-804.

[72] Kalin NH SS, Davidson RJ: Role of the primate orbitofrontal cortex in mediating anxious temperament. Biol Psychiatry 2007, 62:1134-1139.

[73] Davidson RJ MJ, Tomarken AJ, Henriques JB: While a phobic waits: regional brain electrical and autonomic activity in social phobics during anticipation of public speaking. Biol Psychiatry 2000, 47:85-95.

[74] Fischer H AJ, Furmark T, Wik G, Fredrikson M: Right-sided human prefrontal brain activation during acquisition of conditioned fear. Emotion 2002, 2:233-241.

[75] Baas J G, C., Pine, D., Nugent, M., Drevets, W., \& Furey, M.: Prefrontal cortex activation during phasic fear and sustained anxiety to predictable (signaled) and unpredictable (non-signaled) shock. NeuroImage 2004, 22:S26.

[76] Dalton KM KN, Grist TM, Davidson RJ: Neural-cardiac coupling in threat-evoked anxiety. J Cogn Neurosci 2005, 17:969-980

[77] Tranel D BA, Denburg NL: Asymmetric functional roles of right and left ventromedial prefrontal cortices in social conduct, decision-making, and emotional processing. Cortex 2002, 38:589-612.

[78] Clark L MF, Antoun N, Sahakian BJ, Robbins TW: The contributions of lesion laterality and lesion volume to decision-making impairment following frontal lobe damage. Neuropsychologia 2003, 41:1474-1483.

[79] Murphy FC N-SI, Lawrence AD: Functional neuroanatomy of emotions: a meta-analysis. Cogn Affect Behav Neurosci 2003, 3:207-233.

[80] Saletu B AP, Saletu-Zyhlarz GM, Gruber D, Metka M, Huber J: Identifying target regions for vigilance improvement under hormone replacement therapy in postmenopausal syndrome patients by means of electroencephalographic tomography
(LORETA). Psychopharmacology (Berl) 2005, 178:389-399.

[81] Bradley BP MK, Lee SC: Attentional biases for negative information in induced and naturally occurring dysphoria. Behav Res Ther 1997, 35:911-927.

[82] Simmons A SI, Matthews SC, Paulus MP, Stein MB: Anticipation of aversive visual stimuli is associated with increased insula activation in anxiety-prone subjects. Biol Psychiatry 2006, 60:402-409.

[83] Aron AR MS, Sahakian BJ, Robbins TW A componential analysis of task-switching deficits associated with lesions of left and right frontal cortex. Brain 2004, 127:1561-1573.

[84] Forstmann BU JS, Scholte HS, Wolfensteller U, van den Wildenberg WP, Ridderinkhof KR: Function and structure of the right inferior frontal cortex predict individual differences in response inhibition: a model-based approach. J Neurosci 2008a, 28:9790-9796.

[85] Goldin PR MK, Ramel W, Gross JJ: The neural bases of emotion regulation: reappraisal and suppression of negative emotion. Biol Psychiatry 2008, 63:577-586.

[86] Forstmann BU vdWW, Ridderinkhof KR: Neural mechanisms, temporal dynamics, and individual differences in interference control. J Cogn Neurosci 2008b, 20:1854-1865.

[87] Slack SE PS, McMahon SB, Thompson SW, Malcangio M: Brain-derived neurotrophic factor induces NMDA receptor subunit one phosphorylation via ERK and PKC in the rat spinal cord. Eur J Neurosci 2004, 20:1769-1778.

[88] Pandey SC ZH, Roy A, Misra K: Central and medial amygdaloid brain-derived neurotrophic factor signaling plays a critical role in alcohol-drinking and anxiety-like behaviors. J Neurosci 2006, 26:8320-8331

[89] Simmons A MS, Stein MB, Paulus MP: Anticipation of emotionally aversive visual stimuli activates right insula. Neuroreport 2004, 15:2261-2265.

[90] Critchley HD CD, Chandler MP, Mathias CJ, Dolan RJ: Cerebral correlates of autonomic cardiovascular arousal: a functional neuroimaging investigation in humans. J Physiol 2000, 523 Pt 1:259-270.

[91] Colivicchi F BA, Santini M, Caltagirone C: Cardiac autonomic derangement and arrhythmias in right-sided stroke with insular involvement. Stroke 2004, 35:2094-2098.

[92] Thiel CM SR: Dopaminergic lateralisation in the forebrain: relations to behavioural asymmetries and anxiety in male Wistar rats. Neuropsychobiology 2001, 43:192-199.

[93] AD C: Forebrain emotional asymmetry: a neuroanatomical basis? Trends Cogn Sci 2005, 9:566-571.

[94] Saleh TM CB, Cribb AE Sympathoexcitatory effects of estrogen in the insular cortex are mediated by GABA. Brain Res 2005, 1037:114-122.

[95] Kimmerly DS OLD, Menon RS, Gati JS, Shoemaker JK: Cortical regions associated with autonomic cardiovascular regulation during lower body negative pressure in humans. J Physiol 2005, 569:331-345. 